\title{
Semi-infinite Domain Artificial Boundary Conditions in Dynamic Analysis
}

\author{
Ye-Dan ${ }^{1, a}$, Sun-Yijia ${ }^{2, b}$, Li-Yawei $^{3, c}$ and Guo-kexing ${ }^{4, d}$ \\ ${ }^{1}$ School of Civil Engineering, Chang'an University, Xi'an 710061, China \\ ${ }^{2}$ School of Civil Engineering, Chang'an University, Xi'an 710061, China \\ 3China Construction First Group The Second Construction Co.,Ltd.,Beijing 102600,China; \\ 4Tianjin Cement Industry Design and Research Institute Co,Ltd.,Tianjin 300400,China; \\ a1175735451@qq.com, bemail1404894005@qq.com, 'email980311772@qq.com
}

Keywords: numerical simulation; semi-infinite domains; viscoelastic boundary; infinite element boundary.

\begin{abstract}
The problem of wave propagation in a semi-infinite medium and its interaction with the structure was always encountered in the engineering numerical simulation.Artificial boundary conditions of truncated place in semi-infinite domain is the key to ensure accuracy of the calculation results.Adopting fixed boundary hypothesis makes system become a closed system, which clearly contrary to purpose of setting the artificial boundary conditions.Based on ABAQUS software,fixed boundary,viscous boundary, viscoelastic boundary, infinite element boundary and remoted boundary were set respectively,to study vibration response of the simple semi-infinite domain. Results show that under the condition of simple elastic medium,viscoelastic boundary model can get close to the results of actual situation, and also has the advantages of the simple operation, high precision and good stability.
\end{abstract}

\section{Introduction}

When the finite element method is used to solve wave scattering problems in earthquake engineering, the finite computational area should be truncated from the semi-infinite space. The method that cut off the boundary is one of the most direct and efficient technique for modeling a semi-infinte far field in the vibration response analysis of subway station,tunnel and other underground structures. Using simple fixed boundary condition, will makes the whole system is closed and the pulse wave will be reciprocating reflection on the fixed boundary,causing the distortion of simulation ${ }^{[1]}$.

In recent years, in order to eliminate the wave reflection,there have been lots of related scholars come up with a variety of artificial boundary conditions, such as the remoted boundary,the infinite element artificial boundary, the viscous-spring artificial boundary, paraxial approximation boundary and transmitting boundary, etc ${ }^{[2,3]}$.

Based on ABAQUS software,fixed boundary, viscous boundary, viscoelastic boundary , infinite element boundary and remoted boundary were set respectively,to study vibration response of the simple semi-infinite domain.Finally,the viscoelastic boundary model method was verified that the viscoelastic boundary has multiple advantages such as being simple technology processing, low computational cost and high precision,.

\section{The boundary conditions}

\subsection{Remoted boundary condition}

When considering the spread of the infinite domain wave, the boundary of the transmission medium is infinite.But restricted by calculation in the actual amount of calculation, we have to artificial truncate infinite domain to establish finite element model.Using remoted boundary condition when simulating the infinite domain,the artificial boundary needs to get far away,to make the reflected wave not reach the near field area in the study time which was studied,and to reduce the 
reflected wave disturbance to the structural dynamic response.So remoted boundary distance should satisfy (1) ${ }^{[4]}$.

$$
\mathrm{D} \geq 0.5 \mathrm{vt}
$$

Where D represents distance of border to observation point,v represents the compression wave velocity of soil medium,t represents the record duration of vibration wave.Although amount of calculation of remoted boundary model is large,but remoted boundary has advantages of simple processing technology and high precision compared with the viscoelastic boundary and infinite element boundary.

\subsection{Viscoelastic boundary condition}

Lysmer, first proposed the concept of viscous boundary; Considering accuracy of viscous artificial boundary is not high and has faults such as low frequency drift instability, Liu Jingbo established a two-dimensional artificial viscoelastic boundary on the basis of viscous boundary and cylindrical wave equation $^{[5,6]}$, that is damping and spring seted on the border to absorb energy which radiate outward in the process of system vibration,as shown in figure 1. In polar coordinates, using cylindrical wave hypothesis can deduced the relationship between the velocity and stress or the relationship between the displace and stress in the radius of $\mathrm{R}$ of two-dimensional scattering wave .

$$
\tau(R, t)=-\frac{G}{2 R} \omega(R, t)-\rho C_{s} \frac{\partial \omega(R, t)}{\partial t}
$$

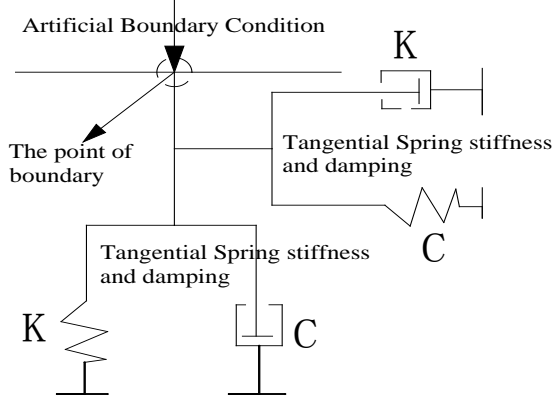

Fig 1 Spring-damper system

where ${ }^{\rho}$ is medium density, c represents the shear wave velocity in the medium,and $\mathrm{G}$ is shear modulus .Spring stiffness and damping of the normal or tangential on the border, which are given as:

$$
K_{B N}=a_{N} \frac{G}{R}, C_{B N}=\rho c_{p} \quad \text { (3) } ; \quad K_{B T}=a_{T} \frac{G}{R}, C_{B T}=\rho c_{s}
$$

Where $\mathrm{K}$ represents spring stiffness ; $\mathrm{G}$ is shear modulus of medium; $\mathrm{R}$ represents the distance from waves to artificial boundary; $C$ is damping coefficient.Lots of research data show that viscoelastic artificial boundary has good convergence. When the a values from 0.35 to 0.65 or from 0.8 to 1.2 , good results can be obtained ${ }^{[7]}$.

\subsection{The infinite element boundary condition}

Concept of infinite element boundary, was put forward by R.ungless ${ }^{[8]}$, which has been widely used in the study of infinite domain through constant development.Damping was introduced in infinite element dynamic analysis theory to absorb radiation energy of wave,so that effect of the wave reflection on the analysis region can be ignored.ABAQUS has considered the damping Settings as above which can be directly use ${ }^{[9]}$.

Infinite element boundary settings in ABAQUS that far point and near point of the infinite element just represent the extension direction of the infinite unit, and its size had no effect on the calculated results of finite element area.Serial number of node in Infinite element should be consistent with entity unit,numbering nodes should press anti-clockwise rules. 

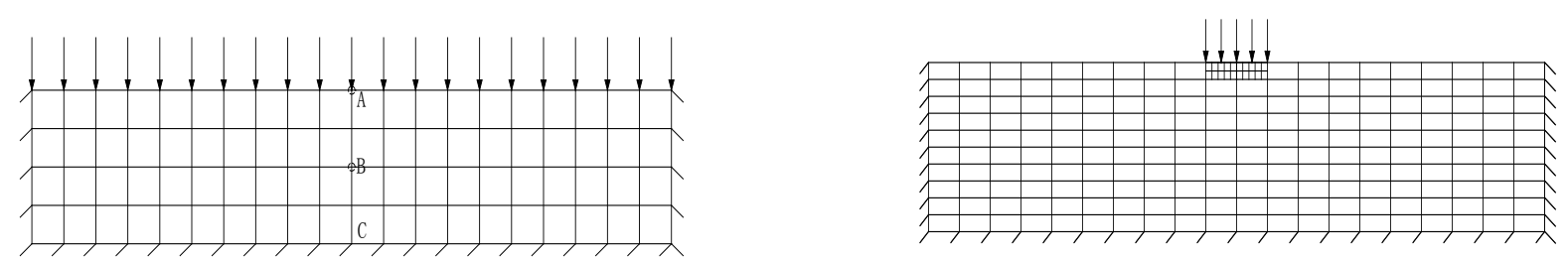

Meanwhile, make ensure that first plane should be the connecting face of finite element and infinite element.As shown in figure 2, the face of the nodes1and 2 should connect with finite element,and the infinite element unit extension direction cannot intersect, As the side 1-3 and 2-4 shown in figure 2 .

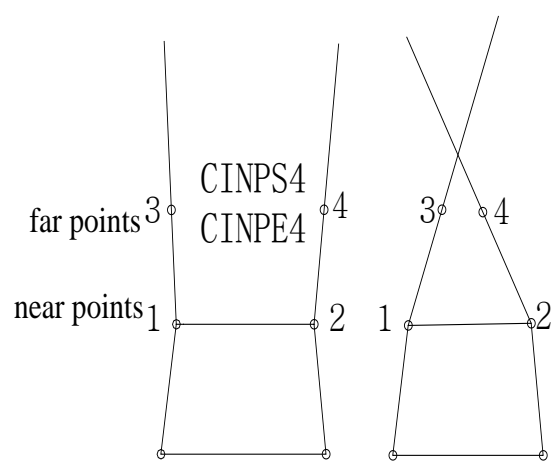

Fig 2 Node layout-error

\section{Example analysis}

3.1 Material indicators of medium

Semi-infinite domains is a single elastic medium.Typical physical and mechanical parameters of soil was selected and finite element analysis model was established in this study,to make the calculation results close to the actual situation.The density of medium is $2 \mathrm{t} / \mathrm{m}^{3}$.Young's modulus is $50000000 \mathrm{~Pa}$; The equivalent wave speed is $250 \mathrm{~m} / \mathrm{s}$; poisson's ratio is 0.3 ; Rayleigh damping a $=$ $0.616, \beta=0.000312$.

\subsection{Numerical analysis model of ABAQUS}

In order to analysis the influence of different artificial boundary conditions to dynamic response analysis of the subway underground structure,to simplify the semi-infinite domains medium to two-dimensional plane strain model.Build a analysis model whose size is $100 \mathrm{~m} * 50 \mathrm{~m}$,mesh size is $1 \mathrm{~m}$.Bottom and side use viscoelastic boundary, viscous boundary and infinite element boundary and fixed boundary one by one.Meanwhile,A remoted boundary model which has $500 \mathrm{~m} * 500 \mathrm{~m}$ size was set,and use its solution as the standard solution.

spring and damping components on the normal and tangential of boudary surfacewas set up lrespectively on boudary surface of viscoelastic boundary mode,distance $\mathrm{R}$ from waves to artificial boundary point was the average distance from point A to bottom $(\mathrm{R}=32 \mathrm{~m})$; Surface of Infinite element boundary model use quadrilateral plane strain unit infinite element to simulate (CINPE4),Its extension points to observation direction which is away from near field;Fixed boundary model with fixed boundary conditions on the ground, the side impose horizontal constraints, the vertical is free.Analysis model of artificial boundary conditions are shown in figure $3 \sim 6$. 


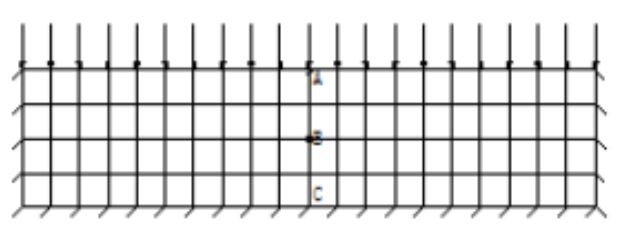

Fig 3 Model of the fixed boundary

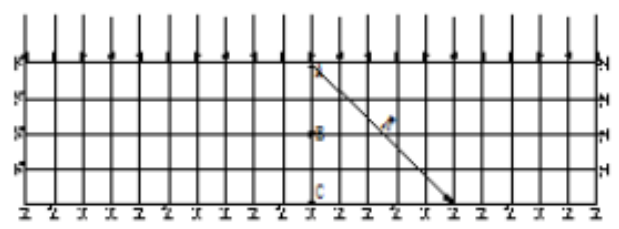

Fig 5 Model of the viscoelastic boundary

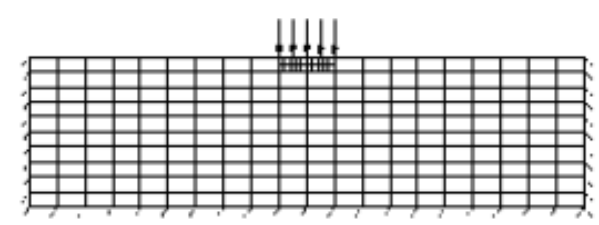

Fig 4 Model of the remoted condition

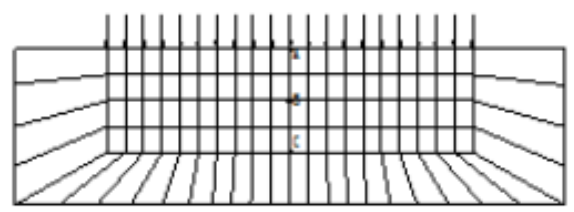

Fig 6 Model of the Infinite element boundary

\subsection{Dynamic response analysis}

On the surface of model applied half sine pulse wave, which time is $0.5 \mathrm{~s}$, amplitude is $0.1 \mathrm{~g}$, load time history is shown in figure 7. When inputing vibration on surface on model,we can not directly input acceleration or velocity, because it make the boundary be equal to the fixed boundary.Release the displacement constraints of bottom boundary which make earthquake input convert into equivalent force input.To sum up, the vertical input no attenuation $P$ wave, the total load for the need to impose ${ }^{[7]}$ :

$$
F_{B N}(t)=-2 \rho c_{p} \dot{u}_{i}
$$

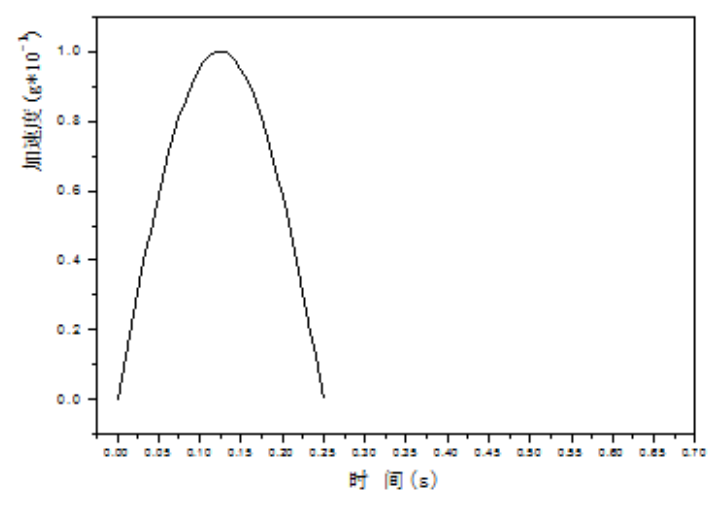

Fig.7 Wave Loading

Vibration response time history figure of observation points A, B and C of each model was selected and analysised,displacement time histories of each point as shown in figure 8. 


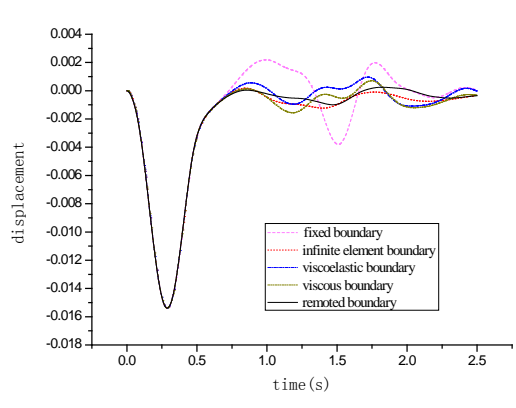

(a) point $\mathrm{A}$

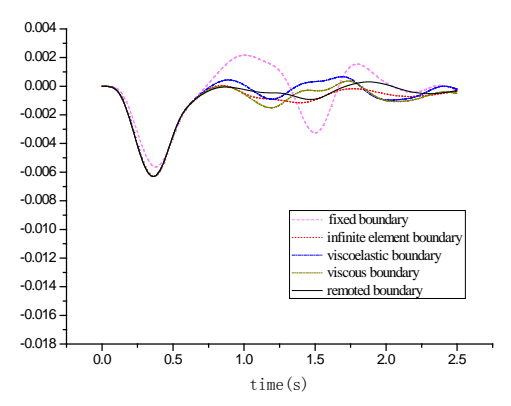

(b) point $B$

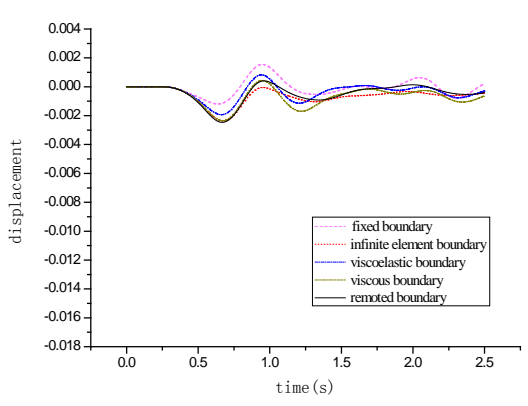

(c) point C

Fig 8 Displacements of observe points

The pulse wave reflected back to top surface and reflected repeatedly without go through the bottom border within the scope of computing and $2.5 \mathrm{~s}$ of computing time under fixed boundary condition.The conclusion that pulse wave always reflected repeatedly without go through the border within the scope of computing and pulse always reflected repeatedly till energy absorbed by the medium where there is damping medium was derivated.Under remoted condition,wave didn't reflected in the calculation time because its model had enough big size and the communication process of wave in medium was consistent with the actual situation in infinite domain as well as accuracy solution.So the conclusion of the remoted boundary condition can be used as a standard solution.

In viscous boundary model, wave energy almost completely absorbed by the viscous boundary in the bottom edge after pulse reached the bottom surface ,and model simulates the wave across the border successfully, but it has the whole drift phenomenon aobviously. Low frequency drift is the fault of viscous boundary, which limits its scope of application.

The conclusion that boundary played the role of consumpting and truncating boundary energy, reducing interference of wave reciprocating and simulating the wave across the boundary without low frequency drift was knew after studying the viscoelastic boundary model and infinite element boundary model. But the results of viscoelastic boundary was far closer to remoted boundary and had a higher accuracy compared with infinite element boundary.

From the calculation results of contrast analysis of above five kinds of different artificial boundary condition model shows that reflected wave had serious interference on calculation results when treated with fixed boundary $n$ the infinite domain truncation surface; Viscoelastic boundary exists the disadvantage of low frequency drift;Viscoelastic boundary and infinite element boundary can better simulate the spread characteristic of the input wave in the infinite domain and they are more convenient to model, easier to implement in ABAQUS.

\section{The result analysis}

The simple elastic medium in semi-infinite domainwas set as the research object, based on nonlinear finite element analysis software platform of ABAQUS ,the semi-infinite domain numerical simulation in the choice of artificial boundary was studied. Dynamic response of different artificial boundary condition model has carried on the contrast research through numerical simulation.draw these conclusions:

(1) Numerical example results show that pulse wave reflected repeatedly in the truncated area which has serious interference on results when the fixed boundary are adopted to simulate the infinite domain medium to truncated boundary in the analysis of the dynamic responses in the semi-infinite domain,so the results of numerical simulation is not good.

(2) Using remoted boundary condition to simulate semi-infinite domain under the conditions of boundary distance is greater than the reflectionless critical value and reflection waves didn't reach the near field area on the time of analysis can reduce adverse effect of reflection interference and make numerical simulation result accord with the wave propagation in actual situation of the infinite 
domain ;But when the calculation of remoted boundary condition model is large,remoted boundary condition model isn't suitable for implementing because of its high calculating cost.

(3) Using viscous boundary exists the phenomenon of low frequency instability, its use is restricted.

(4) Using viscoelastic boundary and infinite element boundary conditions, can reduce the wave reflection, to simulate the wave propagation in the infinite domain media reality, finally get close to results of remoted boundary condition. Also found that compared with the infinite element boundaries, viscoelastic boundary far closer to analysis results of the remoted boudary,and has good precision as well as the more simple modeling work.

\section{References}

[1] Cheng Guoxing, Geotechnical earthquake engineering [M]. Beijing: science Press, 2007:554-563.

[2] Dominguez J.Boundary elements in dynamics[M].Southampton:Computational Mechanics Publications, 1993.

[3] Sun Haifeng, Jing Liping, Men Xianchun, et al. Wang Weining. The selection of boundary conditions in dynamic problems by using ABAQUS [J]. Journal of Earthquake Engineering and Engineering Vibration, 2011,31(3):71-76.

[4] Lou Menglin, Pan Danguang, Fan Lichu. Effect of Vertical Boundary on Seismic Response of Soil layer [J]. Journal of Tongji university (NATURAL SCIENCE), 2003, 31(07):757-761.

[5] Deeks A J, Randolph M F. Axisymmetric time-domaintransmitting boundaries [J]. Journal of EngineeringMechanics, ASCE, 1994, 120(1):25 42.

[6] Liu Jingbo, Du Yixing, Yan Qiushi. The implementation of viscoelastic artificial boundary and the ground motion input in the general finite element software [J]. Journal of Disaster Prevention and Mitigation Engineering, 2007, 27(4):37-42.

[7] UNGLESS R F . An infinite finite element[R].Vancouver : University of British Columbia,1973.

[8] Fei Kang, Zhang Jiangwei. The application of ABAQUS in geotechnical engineering [M]. Beijing: China Water Power Press, 2010:53-60.

[9] Li Xiuqiang, Jiang Tong, Yue Jianyong, et al. Application of Infinite Element Boundary in the Analysis of Subway-Induced Environment Vibration [J]. Chinese Journal of Underground Space and Engineering, 2011,1(7):1377-1383.0 